\title{
Hydroxylation of Phenol Catalyzed by Iron Metal-Organic Framework (Fe-BTC) with Hydrogen Peroxide
}

\author{
Samiran Bhattacharjee*, Mohammad A. Matin \\ Centre for Advanced Research in Sciences (CARS), University of Dhaka, Dhaka, Bangladesh \\ Email: ${ }^{\star}$ s.bhattacharjee@du.ac.bd
}

How to cite this paper: Bhattacharjee, S. and Matin, M.A. (2020) Hydroxylation of Phenol Catalyzed by Iron Metal-Organic Framework (Fe-BTC) with Hydrogen Peroxide. Journal of Materials Science and Chemical Engineering, 8, 55-64.

https://doi.org/10.4236/msce.2020.82006

Received: January 9, 2020

Accepted: February 8, 2020

Published: February 11, 2020

Copyright () 2020 by author(s) and Scientific Research Publishing Inc. This work is licensed under the Creative Commons Attribution-NonCommercial International License (CC BY-NC 4.0).

http://creativecommons.org/licenses/by-nc/4.0/

\begin{abstract}
Liquid phase catalytic hydroxylation of phenol by Fe-containing metal-organic framework, Fe-BTC (BTC = 1,3,5-benzenetricarboxylate) using $30 \% \mathrm{H}_{2} \mathrm{O}_{2}$ as an oxidant and $\mathrm{H}_{2} \mathrm{O}$ as solvent showed good activity and stability under mild reaction conditions. Phenol reacts with hydrogen peroxide over Fe-BTC to produce two main products, viz., catechol and hydroquinone. The effect of temperature, time, substrate/hydrogen peroxide mole ratio and amount of catalyst on catalytic performance were studied. The catalyst could be reused four times without losing significant loss of catalytic performance. The crystallinity and structure of catalyst were unchanged during the catalysis reaction, as confirmed by comparison of XRD and SEM of the fresh and reused catalyst. A reaction mechanism is proposed based on the experimental results.
\end{abstract}

\section{Keywords}

Metal-Organic Frameworks, Fe-BTC, Phenol Hydroxylation, Hydrogen Peroxide

\section{Introduction}

The hydroxylation of phenol to dihydroxybenzenes is an important organic intermediate for the synthesis of perfumes, dyes, medicines, pesticides, rubbers and other fine chemicals [1] [2] [3]. However, phenol is also known as highly toxic, carcinogenic and poorly biodegradable waste from most chemical industries wastewater [4] [5]. From the point of environmental view, the conversion of phenol to value added products is highly desired. The products, catechol (CAT) and hydroquinone (HQ) are synthesized by various soluble oxidizing 
agents [6], which require toxic solvents, high temperature reaction and also generate large quantities of wastes [7]. In recent years, much attention has been dedicated on the development of selective, reusable and stable heterogeneous catalysts for the direct one-step selective hydroxylation of phenol to corresponding catechol (CAT) and hydroquinone (HQ) using hydrogen peroxide as an oxidant due to cheap and environmentally benign reagent which produces only water and molecular oxygen as by-product during catalysis reaction [8]-[13].

Metal-organic frameworks (MOFs), as a new class of crystalline porous materials have lots of potential applications in catalysis [14] [15] [16] [17] due to large surface area, ordered crystalline structure, ability to tune pore size, structural diversity and chemical stability. Fe-containing MOFs, iron metal-organic frameworks of 1,3,5-benzenetricarboxylate (BTC), Fe-BTC have been appeared to be an active catalyst for a large variety of organic synthesis [18] [19] [20] [21] [22]. This MOF, commercialized as Basolite F300, has a semiamorphous structure with specific surface area of $1300-1600 \mathrm{~m}^{2} / \mathrm{g}$ and large pore size. The framework of Fe-BTC is composed of trimers of iron octahedral sharing a common vertex $\mu_{3}-\mathrm{O}$ connected by the bridging organic linker (BTC), which exhibits two types of mesoporous cages of free apertures of 25 and $29 \AA$, accessible through two types of microporous windows of 5.5 and $8.6 \AA$ [18] [21] [23] [24]. In particular, another crystalline Fe-based MOF, MIL-100(Fe) is iso-structural to Fe-BTC, having the same building units with two types of cages in the range of mesopores [25]. The catalytic behavior of both MOFs has been systematically compared in various catalysis reactions to find out the relation between structure and activity [26] [27] [28] [29]. Wang et al. reported the catalytic activity of two Fe-based MOFs, MIL-100(Fe) and MIL-68(Fe), for the photo-catalytic benzene hydroxylation to phenol using $30 \% \mathrm{H}_{2} \mathrm{O}_{2}$ in acetonitrile that showed high selectivity [30]. Recently, we have reported the synthesis of new Fe-containing MOF, Fe-MOF-74, having identical framework structure for previously reported for MOF-74, which showed higher product yield (CAT + HQ) as well as a shorter induction period than other iron based catalysts such as Fe-MCM-41 or $\mathrm{FeO}_{\mathrm{x}} / \mathrm{MCM}-41$ for phenol hydroxylation using hydrogen peroxide in water [7]. As a consequence, very recently, we have also reported that iron-based MOF, Fe-BTC shows high selectivity and stability in the hydroxylation of benzene and toluene using $30 \% \mathrm{H}_{2} \mathrm{O}_{2}$ [31]. These results encouraged us to test the catalytic performance of $\mathrm{Fe}$-containing commercially available $\mathrm{MOF}$ as catalyst for hydroxylation of arenes using $\mathrm{H}_{2} \mathrm{O}_{2}$, since $\mathrm{H}_{2} \mathrm{O}_{2}$ is cheap and environmentally friendly reagent as well as easy catalyst recovery from reaction mixture from ecological point of view. In the present study, we describe the catalytic efficacy of commercially available iron-containing MOF, Fe-BTC, as selective, reusable and stable catalyst for hydroxylation of phenol for the preparation of commercially important diphenols using $\mathrm{H}_{2} \mathrm{O}_{2}$. The effect of various reaction parameters, such as, temperature, time, substrate-oxidant mole ratio and catalyst amount on catalytic performance were investigated. This work describes in more detail, the sta- 
bility of Fe-BTC using various spectroscopic and microscopic techniques.

\section{Experimental}

\subsection{Materials}

Phenol ACS reagent (99\%), hydrogen peroxide (30\%) and MOF (Fe-BTC, Basolite ${ }^{\oplus}$ 300) were obtained from Sigma-Aldrich and used without further treatment.

\subsection{Characterization of Fe-BTC}

Powder X-ray diffraction pattern was recorded on a RigakuUltimaIVdiffractometer with $\mathrm{Cu}-\mathrm{K} \alpha(\lambda=1.54 \AA)$ at $0.2 \mathrm{~min}^{-1}$. FTIR spectra were measured as $\mathrm{KBr}$ discs on aIRPrestige (Shimadzu) spectrometer. SEM micrographs were taken on a JEOL (JSM-6490LA) instrument.

\subsection{Catalysis Reaction}

The hydroxylation of phenol was performed in a two-necked round bottom flask fitted with a water-cooled condenser. Typically, phenol $(470 \mathrm{mg})$, water $\left(15 \mathrm{~cm}^{3}\right)$ and catalyst $(20 \mathrm{mg})$ were introduced to a round bottom flask and the mixture stirred at $50^{\circ} \mathrm{C}$. Required amount of hydrogen peroxide (30\%) was added into the reaction mixture through a syringe with constant stirring. The conversion and products were analyzed by GC (Perkin-Elmer, Clarus 500) fitted with a DB-Wax coated capillary column $(30 \mathrm{~m}, 0.32 \mathrm{~mm}, 0.25 \mu \mathrm{m})$ and an FID. The products were identified by comparison with that of the authentic compounds. The concentration of phenol was determined from the average three runs.

\section{Results and Discussion}

The hydroxylation of phenol was studied in water medium using $30 \% \mathrm{H}_{2} \mathrm{O}_{2}$ over Fe-BTC by varying the various reaction parameters, such as temperature, time, substrate $/ \mathrm{H}_{2} \mathrm{O}_{2}$ mole ratio and amount of catalyst. Phenol was converted to two main products, such as, catechol (CA) and hydroquinone (HQ). The reaction is shown in Scheme 1.

The influence of temperature on the catalytic performance of phenol hydroxylation is shown in Figure 1. Phenol conversion increases from 8.8\% to $16.9 \%$ with gradually increasing the reaction temperature from $35^{\circ} \mathrm{C}$ to $70^{\circ} \mathrm{C}$ using<smiles>O=C1C=CC(=O)C=C1</smiles>

Scheme 1. Phenol hydroxylation reaction catalyzed over Fe-BTC. 


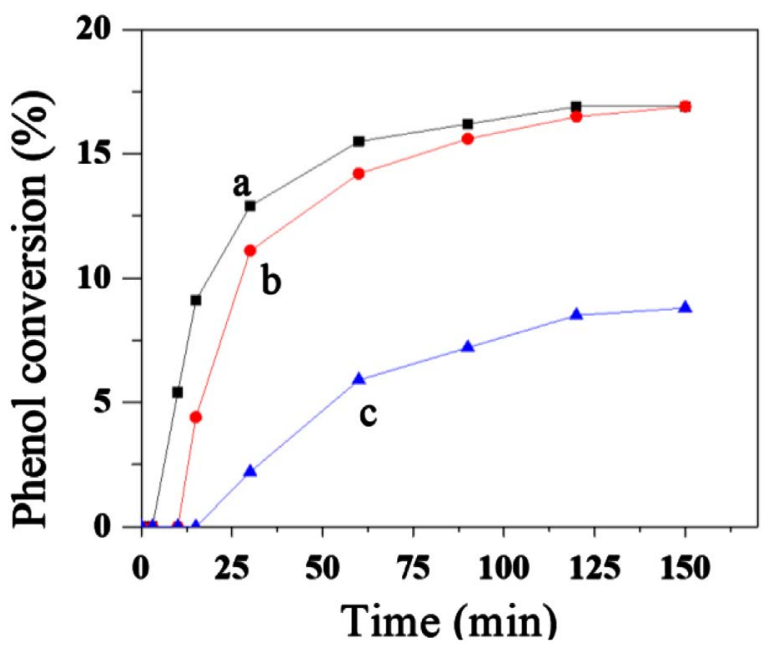

Figure 1. Effect of reaction temperature on the catalytic activity over Fe-BTC for phenol hydroxylation of phenol with phenol: $\mathrm{H}_{2} \mathrm{O}_{2}$ ratio of $1: 1$ : (a) $70^{\circ} \mathrm{C}$, (b) $50^{\circ} \mathrm{C}$ and (c) $35^{\circ} \mathrm{C}$.

phenol to hydrogen peroxide mole ratio of 1:1, however, the induction period became shorter with an increase of temperature (Table 1). On the other hand, BQ selectivity increases from $2.3 \%$ to $8.2 \%$ with increase of temperature due to further oxidation of $\mathrm{HQ}$ to $\mathrm{BQ}$ [7].

Figure 2 shows the conversion of phenol as a function of hydrogen peroxide concentration with varying the amount of $\mathrm{H}_{2} \mathrm{O}_{2}$ from 1: 0.5 to $1: 2$ in phenol to $\mathrm{H}_{2} \mathrm{O}_{2}$ mole ratio at $50^{\circ} \mathrm{C}$. Phenol conversion increased from $9.1 \%$ to $16.9 \%$ with an increase in the concentration of $\mathrm{H}_{2} \mathrm{O}_{2}$ from 1:0.5 to 1:1 in phenol to $\mathrm{H}_{2} \mathrm{O}_{2}$ mole ratio and then leveled off to $16.9 \%$.

To examine the effect of catalyst amount on the catalytic activity the phenol hydroxylation reaction was carried out with varying the amount of catalyst from $0.010 \mathrm{~g}$ to $0.030 \mathrm{~g}$ using phenol to $\mathrm{H}_{2} \mathrm{O}_{2}$ mole ratio of $1: 1$ at $50^{\circ} \mathrm{C}$. As shown in Table 1, the phenol conversion was not effected by increasing the catalyst amount and only the induction period became shorter with increasing the amount of Fe-BTC.

Figure 3 shows the reaction profile as a function of reaction time. The reaction was carried out using phenol to $\mathrm{H}_{2} \mathrm{O}_{2}$ mole ratio of $1: 1$ at $50^{\circ} \mathrm{C}$. The results showed that the conversion increased progressively with time, while the selectivity of CA remained fairly constant at ca. $66.9 \%$, but, the selectivity of HQ was largely affected with a drop from $32.4 \%$ to $27.9 \%$. During this period, BQ selectivity increased from $0.2 \%$ to $5.2 \%$. This may be due to further oxidation of $\mathrm{HQ}$ to $\mathrm{BQ}$ in the presence of excess $\mathrm{H}_{2} \mathrm{O}_{2}$ [7].

Based on experimental data, a proposed reaction mechanism is shown in Scheme 2. The reaction proceeds through the formation of hydroxyl radical via $\mathrm{Fe}^{2+} / \mathrm{Fe}^{3+}$ in the presence of $\mathrm{H}_{2} \mathrm{O}_{2}$ and catalyst, and finally the attack of hydroxyl radicals at ortho or para positions of phenol to generate CAT and HQ [7] [32]. Hydroquinone can be further oxidized to benzoquinone (BQ). 
Table 1. Influence of reaction temperature, phenol/ $\mathrm{H}_{2} \mathrm{O}_{2}$ mole ratio and amount of catalyst on the hydroxylation of phenol over Fe-BTC.

\begin{tabular}{|c|c|c|c|c|c|c|c|}
\hline \multirow{2}{*}{$\begin{array}{c}\text { Temp. } \\
\left({ }^{\circ} \mathrm{C}\right)\end{array}$} & \multirow{2}{*}{ Phenol: $\mathrm{H}_{2} \mathrm{O}_{2}$} & \multirow{2}{*}{$\begin{array}{l}\text { Catalyst } \\
\text { amount } \\
\text { (g) }\end{array}$} & \multirow{2}{*}{$\begin{array}{c}\text { Induction } \\
\text { period (min) }\end{array}$} & \multirow{2}{*}{$\begin{array}{c}\text { Phenol } \\
\text { conv. } \\
(\%)\end{array}$} & \multicolumn{3}{|c|}{ Product selectivity (\%) } \\
\hline & & & & & CAT & HQ & BQ \\
\hline 35 & $1: 1$ & 0.020 & 15 & 8.8 & 66.6 & 31.1 & 2.3 \\
\hline \multirow[t]{8}{*}{50} & $1: 0.50$ & 0.020 & 14 & 9.1 & 66.6 & 31.3 & 2.1 \\
\hline & $1: 1$ & 0.020 & 10 & 16.9 & 66.9 & 27.9 & 5.2 \\
\hline & $1: 2$ & 0.020 & 4 & 17.0 & 66.4 & 25.9 & 7.7 \\
\hline & $1: 1$ & 0.010 & 16 & 16.0 & 66.6 & 28.0 & 5.4 \\
\hline & $1: 1^{\mathrm{a}}$ & 0.020 & 10 & 16.9 & 66.9 & 27.9 & 5.2 \\
\hline & $1: 1$ & 0.030 & 3 & 16.9 & 66.5 & 28.0 & 5.5 \\
\hline & $1: 1^{\mathrm{b}}$ & 0.020 & 10 & 16.9 & 66.9 & 27.9 & 5.2 \\
\hline & $1: 1^{\mathrm{c}}$ & 0.020 & 10 & 16.9 & 66.9 & 27.9 & 5.2 \\
\hline 70 & $1: 1$ & 0.020 & 3 & 16.9 & 66.4 & 25.4 & 8.2 \\
\hline
\end{tabular}

${ }^{\mathrm{a}}$ First run. ${ }^{\mathrm{b}}$ Second run. ${ }^{\mathrm{c}}$ Fourth run.

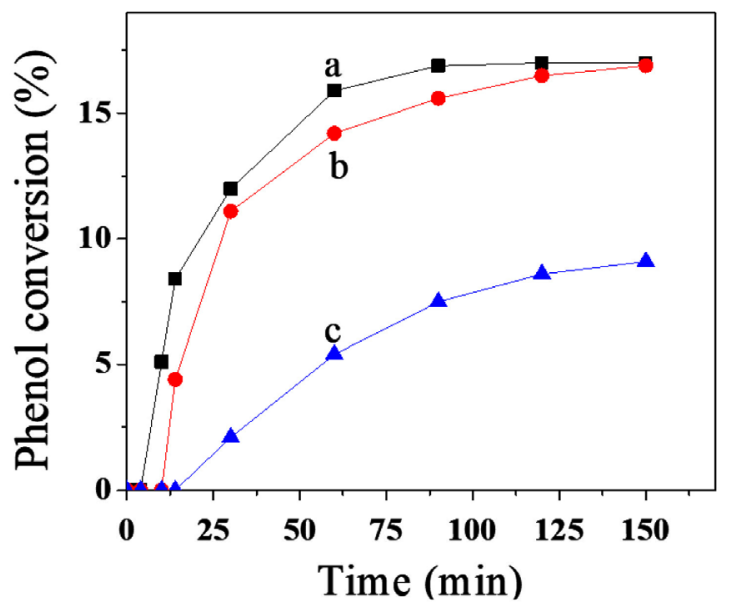

Figure 2. Effect of phenol to $\mathrm{H}_{2} \mathrm{O}_{2}$ ratio for phenol hydroxylation at $50^{\circ} \mathrm{C}$ : (a) $1: 2$, (b) $1: 1$ and (c) 1:0.5.

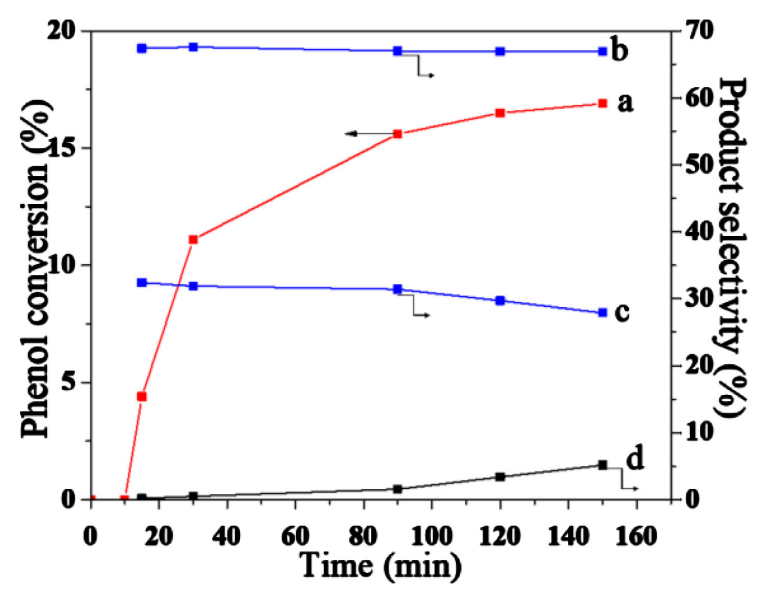

Figure 3. Effect of reaction time on the activity over Fe-BTC for phenol hydroxylation with phenol: $\mathrm{H}_{2} \mathrm{O}_{2}$ ratio of $1: 1$ at $50^{\circ} \mathrm{C}$ : (a) phenol conversion, (b) CAT selectivity, (c) HQ selectivity and (d) BQ selectivity. 


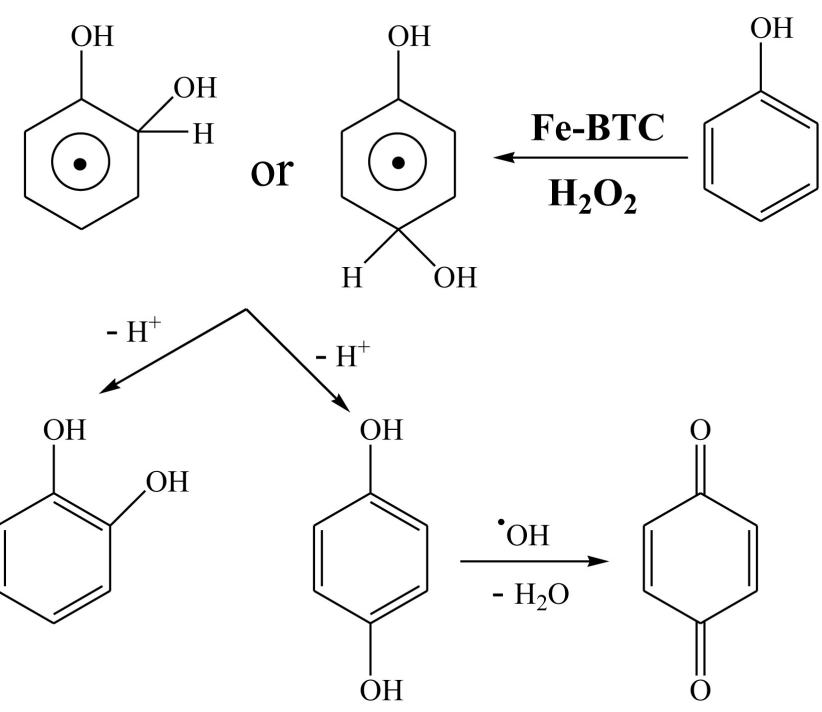

Scheme 2. Reaction path for phenol hydroxylation reaction catalyzed over Fe-BTC.

It is important to check the stability of the catalyst under the same reaction conditions, since active metal leached from the catalyst could be responsible for the experimental catalytic performance [33] [34]. The stability of catalyst in phenol hydroxylation was examined by performing consecutive reuses of Fe-BTC using phenol-to- $\mathrm{H}_{2} \mathrm{O}_{2}$ mole ratio of $1: 1$ at $50^{\circ} \mathrm{C}$. At the end of each reaction cycle, Fe-BTC was recovered by simple filtration and washed with water and ethanol, and dried at $120^{\circ} \mathrm{C}$ under vacuum for $4 \mathrm{~h}$ and reused. The catalyst was reusable for four times with little loss of activity and selectivity (Table 1). XRD of reused catalyst indicated that the structure of catalyst was retained after recycle run (Figure 4). The reused catalyst was also examined by scanning electron microscope (SEM). The SEM images confirmed that the morphology was retained during the reaction (Figure 5). These observations support that the phenol hydroxylation reaction took place at the framework Fe sites within the MOF matrix.

\section{Conclusion}

The commercially available MOF, Fe-BTC showed an efficient heterogeneous catalyst for hydroxylation of phenol to useful products such as catechol (CAT) and hydroquinone (HQ) using environmentally benign $30 \%$ hydrogen peroxide in water medium under mild reaction conditions. The material could be recycled without significant loss of catalytic activity and product selectivity. In addition, commercially available, eco-friendly route and high stability of Fe-BTC catalyst during catalysis reaction in hydroxylation of phenol make this catalytic system attractive for industrial application for diphenol production. The formation of hydroxyl radical through redox path over the Fe-BTC catalyst and the attack of hydroxyl radical at ortho or para position of phenol was proposed as the mechanism for hydroxylation of phenol. 


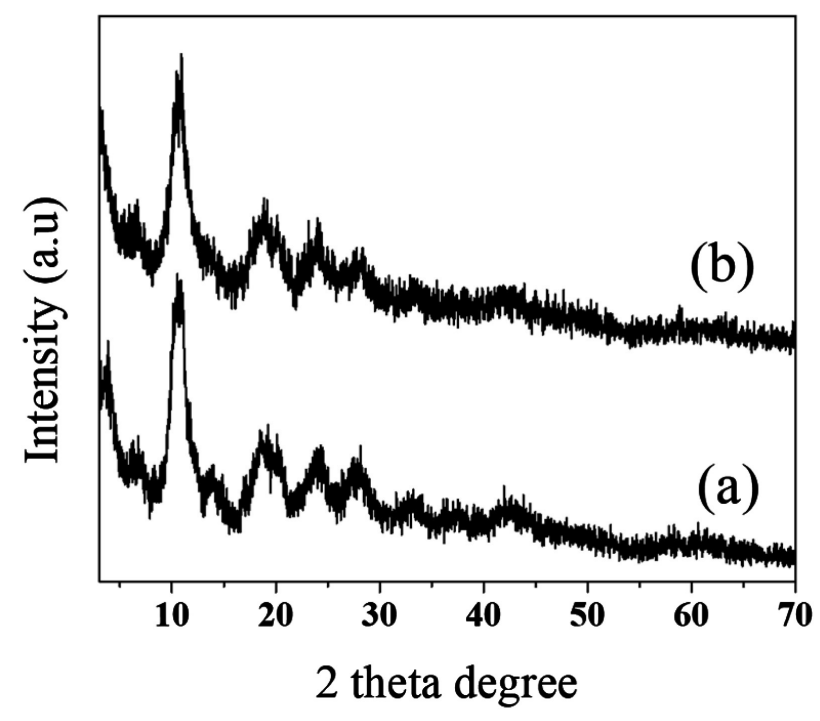

Figure 4. X-ray powder diffraction patterns of (a) Fe-BTC and (b) reused Fe-BTC catalyst after fourth run.
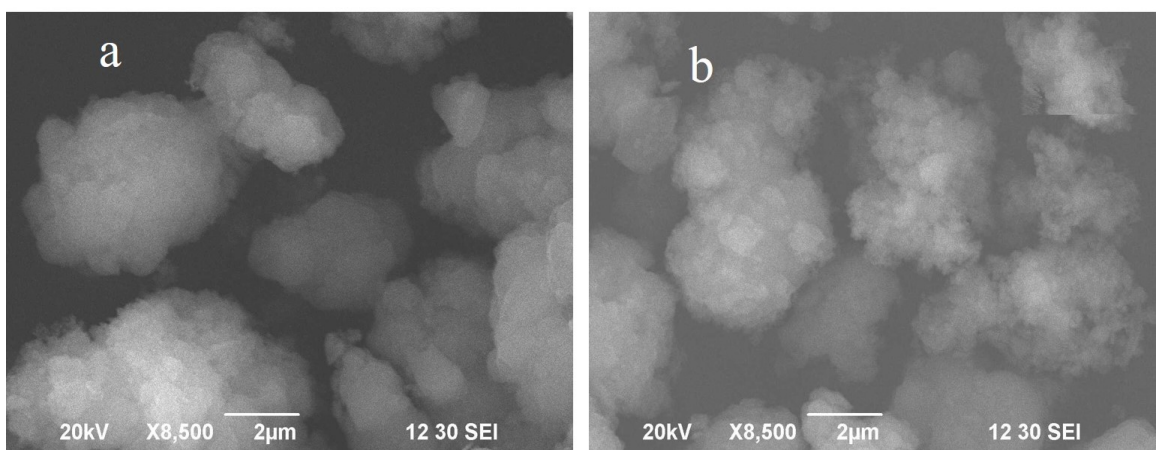

Figure 5. Scanning electron microscope of (a) fresh Fe-BTC and (b) reused Fe-BTC catalyst after fourth run. $20 \mathrm{kV}$ accelerating voltage, the X8500 current magnification and 2 $\mu \mathrm{m}$ scale bar were applied in this scanning.

\section{Acknowledgements}

Financial support of this work by the Centre for Advanced Research in Sciences (University of Dhaka, Dhaka, Bangladesh) is greatly appreciated.

\section{Conflicts of Interest}

The authors declare no conflicts of interest regarding the publication of this paper.

\section{References}

[1] Wang, J., Park, J.N., Wei, X.Y. and Lee, C.W. (2003) Room-Temperature Heterogeneous Hydroxylation of Phenol with Hydrogen Peroxide over $\mathrm{Fe}^{2+}, \mathrm{Co}^{2+}$ Ion-Exchanged $\mathrm{Na} \beta$ Zeolite. Chemical Communications, 5, 628-629. https://doi.org/10.1039/b212296k

[2] Ikurumi, S., Okada, S., Nakatsuka, K., Kamegawa, T., Mori, K. and Yamashita, H. (2014) Enhanced Activity and Selectivity in the One-Pot Hydroxylation of Phenol 
by $\mathrm{Pd} / \mathrm{SiO}_{2} @ \mathrm{Fe}$-Containing Mesoporous Silica Core-Shell Catalyst. The Journal of Physical Chemistry C, 118, 575-581. https://doi.org/10.1021/jp411153p

[3] Wu, G.Q., Xiao, J.H., Zhang, L., Wang, W.J., Hong, Y.Q., Huang, H.J., Jiang, Y., Lia, L. and Wang, C.R. (2016) Copper-Modified TS-1 Catalyzed Hydroxylation of Phenol with Hydrogen Peroxide as the Oxidant. RSC Advances, 6, 101071-101078. https://doi.org/10.1039/C6RA20980G

[4] Santos, A., Yustos, P., Quintanilla, A., Rodriguez, S. and Garcia-Ochoa, F. (2002) Route of the Catalytic Oxidation of Phenol in Aqueous Phase. Applied Catalysis B: Environmental, 39, 97-113. https://doi.org/10.1016/S0926-3373(02)00087-5

[5] Guyer, H.H. (1998) Industrial Processes and Waste Stream Management. Wiley, New York.

[6] Sheldon, R.A. and Van Santen, R.A. (1995) Catalytic Oxidation: Principal and Applications. World Scientific, Singapore, 79.

[7] Bhattacharjee, S., Choi, J.S., Yang, S.T., Choi, S.B., Kim, J. and Ahn, W.S. (2010) Solvothermal Synthesis of Fe-MOF-74 and Its Catalytic Properties in Phenol Hydroxylation. Journal of Nanoscience and Nanotechnology, 10, 135-141. https://doi.org/10.1166/jnn.2010.1493

[8] Zhang, H., Zhang, X., Ding, Y., Yan, L., Ren, T. and Suo, J. (2002) Hydroxylation of Phenol Catalyzed by Copper Keggin-Type Heteropoly Compounds with Hydrogen Peroxide. New Journal of Chemistry, 26, 376-377. https://doi.org/10.1039/b110574D

[9] Wang, J., Park, J.N., Jeong, H.C., Choi, K.S., Wei, X.Y., Hong, S.I. and Lee, C.W. (2004) $\mathrm{Cu}^{2+}$-Exchanged Zeolites as Catalysts for Phenol Hydroxylation with Hydrogen Peroxide. Energy Fuels, 18, 470-476. https://doi.org/10.1021/ef0300904

[10] Amadine, O., Essamlali, Y., Fihri, A., Larzek, M. and Zahouily, M. (2017) Effect of Calcination Temperature on the Structure and Catalytic Performance of Copper-Ceria Mixed Oxide Catalysts in Phenol Hydroxylation. RSC Advances, 7, 12586-12597. https://doi.org/10.1039/C7RA00734E

[11] Sharma, M., Saikia, G., Ahmed, K., Gogoi, S.R., Puranik, V.G. and Islam, N.S. (2018) Vanadium-Based Polyoxometalate Complex as a New and Efficient Catalyst for Phenol Hydroxylation under Mild Conditions. New Journal of Chemistry, 42, 5142-5152. https://doi.org/10.1039/C7NJ04433J

[12] Luo, G., Jiao, Y., Lv, X., Zhang, X. and Gao, X. (2018) Green Synthesis of Dihydroxybenzene from Phenol with Hydrogen Peroxide Catalyzed by Iron Modified FSM-16. Research on Chemical Intermediates, 44, 5377-5387. https://doi.org/10.1007/s11164-018-3428-7

[13] Amedlous, A., Amadine, O., Essamlali, Y., Daanoun, K., Aadil, M. and Zahouily, M. (2019) Aqueous-Phase Catalytic Hydroxylation of Phenol with $\mathrm{H}_{2} \mathrm{O}_{2}$ by Using a Copper Incorporated Apatite Nanocatalyst. RSC Advances, 9, 14132-14142. https://doi.org/10.1039/C9RA02021G

[14] Bhattacharjee, S., Yang, D.A. and Ahn, W.S. (2011) A New Heterogeneous Catalyst for Epoxidation of Alkenes via One-Step Post-Functionalization of IRMOF-3 with a Manganese(II) Acetylacetonate Complex. Chemical Communications, 47, 3637-3639. https://doi.org/10.1039/c1cc00069a

[15] Bhattacharjee, S., Lee, Y.R. and Ahn, W.S. (2015) Post-Synthesis Functionalization of a Zeoliticimidazolate Structure ZIF-90: A Study on Removal of $\mathrm{Hg}$ (II) from Water and Epoxidation of Alkenes. CrystEngComm, 17, 2575-2582. https://doi.org/10.1039/C4CE02555E 
[16] Zhu, L., Liu, X.Q., Jiang, H.L. and Sun, L.B. (2017) Metal-Organic Frameworks for Heterogeneous Basic Catalysis. Chemical Reviews, 117, 8129-8176. https://doi.org/10.1021/acs.chemrev.7b00091

[17] Huang, Y.B., Liang, J., Wang, X.S. and Cao, R. (2017) Multifunctional Metal-Organic Framework Catalysts: Synergistic Catalysis and Tandem Reactions. Chemical Society Reviews, 46, 126-157. https://doi.org/10.1039/C6CS00250A

[18] Dhakshinamoorthy, A., Alvaro, M. and Garcia, H. (2009) Metal Organic Frameworks as Efficient Heterogeneous Catalysts for the Oxidation of Benzylic Compounds with t-Butylhydroperoxide. Journal of Catalysis, 267, 1-4. https://doi.org/10.1016/j.jcat.2009.08.001

[19] Dhakshinamoorthy, A., Alvaro, M. and Garcia, H. (2010) Aerobic Oxidation of Thiols to Disulfides Using Iron Metal-Organic Frameworks as Solid Redoxcatalysts. Chemical Communications, 46, 6476-6478. https://doi.org/10.1039/c0cc02210a

[20] Dhakshinamoorthy, A., Alvaro, M. and Garcia, H. (2010) Claisen-Schmidt Condensation Catalyzed by Metal-Organic Frameworks. Advanced Synthesis Catalysis, 352, 711-717. https://doi.org/10.1002/adsc.200900747

[21] Dhakshinamoorthy, A., Alvaro, M. and Garcia, H. (2010) Metal-Organic Frameworks as Efficient Heterogeneous Catalysts for the Regioselective Ring Opening of Epoxides. Chemistry: A European Journal, 16, 8530-8536. https://doi.org/10.1002/chem.201000588

[22] Oveisi, A.R., Khorramabadi-zad, A. and Daliran, S. (2016) Iron-Based Metal-Organic Framework, Fe(BTC): An Effective Dual-Functional Catalyst for Oxidative Cyclization of Bisnaphthols and Tandem Synthesis of Quinazolin-4(3H)-Ones. RSC Advances, 6, 1136. https://doi.org/10.1039/C5RA19013D

[23] Youn, H.K., Kim, J. and Ahn, W.S. (2011) MWCNT Synthesis over Fe-BTC as a Catalyst/Carbon Source via CVD. Materials Letters, 65, 3055-3057. https://doi.org/10.1016/j.matlet.2011.06.081

[24] Sciortino, L., Alessi, A., Messina, F., Buscarino, G. and Gelardi, F.M. (2015) Structure of the FeBTC Metal-Organic Framework: A Model Based on the Local Environment Study. The Journal of Physical Chemistry C, 119, 7826-7830. https://doi.org/10.1021/acs.jpcc.5b01336

[25] Horcajada, P., Surblé, S., Serre, C., Hong, D.Y., Seo, Y.K., Chang, J.S., Grenèche, J.M., Mrgiolaki, I. and Férey, G. (2007) Synthesis and Catalytic Properties of MIL-100(Fe), an Iron(III) Carboxylate with Large Pores. Chemical Communications, 27, 2820-2822. https://doi.org/10.1039/B704325B

[26] Dhakshinamoorthy, A., Alvaro, M., Horcajada, P., Gibson, E., Vishnuvarthan, M., Vimont, A., Grenèche, J.M., Serre, C., Daturi, M. and Garcia, H. (2012) Comparison of Porous Iron Trimesates Basolite F300 and MIL-100(Fe) as Heterogeneous Catalysts for Lewis Acid and Oxidation Reactions: Roles of Structural Defects and Stability. ACS Catalysis, 2, 2060-2065. https://doi.org/10.1021/cs300345b

[27] Majano, G., Ingold, O., Yulikov, M., Jeschke, G. and Perez-Ramirez, J. (2013) Room-Temperature Synthesis of Fe-BTC from Layered Iron Hydroxides: The Influence of Precursor Organisation. CrystEngComm, 15, 9885-9892. https://doi.org/10.1039/c3ce41366g

[28] Opanasenko, M., Dhakshinamoorthy, A., Cejka, J. and Garcia, H. (2013) Deactivation Pathways of the Catalytic Activity of Metal-Organic Frameworks in Condensation Reactions. ChemCatChem, 5, 1553-1561. https://doi.org/10.1002/cctc.201200643

[29] Sanchez-Sanchez, M., Asua de, I., Ruano, D. and Diaz, K. (2015) Direct Synthesis, 
Structural Features, and Enhanced Catalytic Activity of the Basolite F300-Like Semiamorphous Fe-BTC Framework. Crystal Growth Design, 15, 4498-4506. https://doi.org/10.1021/acs.cgd.5b00755

[30] Wang, D., Wang, M. and Li, Z. (2015) Fe-Based Metal-Organic Frameworks for Highly Selective Photocatalytic Benzene Hydroxylation to Phenol. ACS Catalysis, 5, 6852-6857. https://doi.org/10.1021/acscatal.5b01949

[31] Bhattacharjee, S. (2018) Hydroxylation of Benzene and Toluene by Heterogeneous Iron Metal-Organic Framework (Fe-BTC). Indian Journal of Chemistry, 57A, 778-783.

[32] Liu, C., Shan, Y., Yang, X., Ye, X. and Wu, Y. (1997) Iron(II)-8-Quinolinol/ MCM-41-Catalyzed Phenol Hydroxylation and Reaction Mechanism. Journal of Catalysis, 168, 35-41. https://doi.org/10.1006/jcat.1997.1612

[33] Lempers, H.E.B. and Sheldon, R.A. (1998) The Stability of Chromium in CrAPO-5, CrAPO-11, and CrS-1 during Liquid Phase Oxidations. Journal of Catalysis, 175, 62-69. https://doi.org/10.1006/jcat.1998.1979

[34] Kim, J., Bhattacharjee, S., Jeong, K.E., Jeong, S.Y., Choi, M., Ryoo, R. and Ahn, W.S. (2010) CrAPO-5 Catalysts Having a Hierarchical Pore Structure for the Selective Oxidation of Tetralin to 1-Tetralone. New Journal of Chemistry, 34, 2971-2978.

https://doi.org/10.1039/c0nj00493f 